\title{
Game Semantics in String Diagrams
}

\author{
Paul-André Melliès \\ CNRS, Laboratoire PPS, UMR 7126 \\ Université Paris Diderot, Sorbonne Paris Cité \\ F-75205 Paris, France
}

\begin{abstract}
A dialogue category is a symmetric monoidal category equipped with a notion of tensorial negation. We establish that the free dialogue category is a category of dialogue games and total innocent strategies. The connection clarifies the algebraic and logical nature of dialogue games, and their intrinsic connection to linear continuations. The proof of the statement is based on an algebraic presentation of dialogue categories inspired by knot theory, and a factorization theorem established by rewriting techniques.
\end{abstract}

Index Terms-Dialogue games, innocent strategies, linear continuations, string diagrams, ribbon categories, coherence theorems, 2 dimensional algebra.

\section{INTRODUCTION}

Tensorial logic. Tensorial logic is a primitive logic of tensor and negation which refines linear logic by relaxing the hypothesis that linear negation is involutive. Its purpose is to resolve in this way the traditional gap between game semantics and linear logic, and to unify both of them with the monadic theory of linear continuations, see [24] for details. The logic emerged from the discovery that the linear distributivity law of linear logic

$$
A \otimes(B \ngtr C) \quad \longrightarrow \quad(A \otimes B)>C
$$

is the "shadow" of a more primitive distributivity law existing in any logic (typically intuitionistic logic) equipped with a notion of conjunction $\otimes$ and of negation. This distributivity law may be written as

$$
A \otimes[B, C] \quad \longrightarrow \quad[A \otimes B, C]
$$

where the $n$-ary connective $\left[A_{1}, \ldots, A_{n}\right]$ is syntactic sugar for

$$
\left[A_{1}, \ldots, A_{n}\right]=\neg\left(\neg A_{1} \otimes \ldots \otimes \neg A_{n}\right) .
$$

This observation was the sparkle which lead to the introduction of tensorial logic, whose sequent calculus is recalled in the Appendix. Interestingly, the distributive law (2) holds in tensorial logic as an instance of a more general distributivity law, defined as follows:

$$
A \otimes\left[B, C_{1}, \ldots, C_{n}\right] \quad \longrightarrow \quad\left[A \otimes B, C_{1}, \ldots, C_{n}\right]
$$

Observe that one recovers in the case $n=0$ the well-known principle which makes the double negation monad a strong monad:

$$
A \otimes \neg \neg B=A \otimes[B] \quad \longrightarrow \quad[A \otimes B]=\neg \neg(A \otimes B) .
$$

These considerations indicate the existence of a unified theory of linear logic and continuations which could benefit from the two approaches. One purpose of the paper is precisely to substantiate this idea, by exhibiting an algebraic presentation by generators and relations of the proofs of tensorial logic. In particular, we will establish in the course of the paper that all the various distributivity laws are generated by the following principle

$$
A \otimes(X \rightarrow B) \quad \longrightarrow \quad X \rightarrow(A \otimes B)
$$

where $X \rightarrow B$ is syntactic sugar for

$$
X \rightarrow B \quad=\quad \neg(X \otimes \neg B)
$$

This work is partly supported by the ANR project RECRE.
This principle (3) should be seen as a parametric variant of the tensorial strength, which is recovered when $X$ is the unit of the tensor product. Note that the principle is provable in tensorial logic by the derivation tree below:

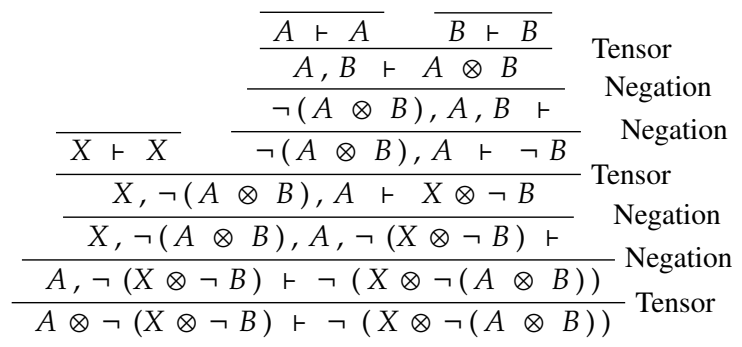

Tensorial proof-nets. It appears that this unified view of linear logic and continuations leads to a compelling notion of proof-net for tensorial logic, which lies at the converging point of:

- multiplicative proof-nets in linear logic,

- innocent strategies in game semantics,

- string diagrams in low dimensional algebra.

In order to devise this graphical notation for tensorial proofs, we start from the algebraic approach to proof theory initiated by Lambek [15] and promoted in his book with Scott [16] using the notion of cartesian-closed category. We briefly recall this notion, and explain how to relate it to string diagrams using ideas coming from knot theory.

Cartesian closed categories. The key idea inspired by Lawvere's categorical semantics is to think of the $\lambda$-calculus as a syntactic presentation of a category with structure. Typically, Lambek established that the free cartesian closed category free-ccc $(\mathscr{X})$ on a given category $\mathscr{X}$ has:

- the formulas of minimal logic (constructed with the binary constructors $\times$ and $\Rightarrow$ together with the conjunctive unit 1) with atoms given by the objects of the category $\mathscr{X}$,

- the simply-typed $\lambda$-terms of type $A \Rightarrow B$ as morphisms from the formula $A$ to the formula $B$, considered modulo a suitable notion of equivalence between $\lambda$-terms (that is, $\beta$ and $\eta$ conversion and composition of maps between atoms in $\mathscr{X}$ ).

This result enables one to construct denotational models of the simply-typed $\lambda$-calculus in a simple and conceptual way. Consider the full and faithful functor

$$
\mathscr{X} \quad \longrightarrow \quad \text { free-ccc }(\mathscr{X})
$$

which transports every object of $\mathscr{X}$ to the corresponding atomic formula in free-ccc $\mathscr{X})$. The theorem established by Lambek states that every functor $\mathscr{X} \longrightarrow \mathscr{D}$ to a cartesian-closed category $\mathscr{D}$ extends to a structure-preserving functor

$$
[-] \quad: \quad \operatorname{free}-\operatorname{ccc}(\mathscr{X}) \quad \longrightarrow \quad \mathscr{D}
$$


making the diagram commute:

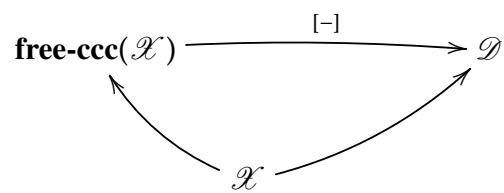

This functor [-] is moreover unique up to structure-preserving isomorphism. The benefit of the construction is that the functor $[-]$ transports every simply-typed $\lambda$-term $P$ to a denotation $[P]$ in the cartesian closed category $\mathscr{D}-$ that is, an invariant of the $\lambda$-term $P$ modulo $\beta \eta$-conversion.

Ribbon categories. This story on cartesian closed categories and intuitionistic logic is well-known among the logicians and the computer scientists interested in the semantics of proofs and programs. What is less known is that the very same story occurs in a quite different part of mathematics - knot theory and representation theory of quantum groups. There, Turaev defined the notion of a ribbon category as a monoidal category equipped with combinators for braiding and Uturns, satisfying a series of expected equations, see for instance [13], [27], [8], [21] for details. Then, one establishes a similar coherence theorem, which states that the free ribbon category on a category $\mathscr{X}$ has

- sequences $\left(A_{1}^{\epsilon_{1}}, \ldots, A_{n}^{\epsilon_{n}}\right)$ of signed objects of $\mathscr{X}$ as objects, where each $A_{i}$ is an object of the category $\mathscr{X}$, and each $\epsilon_{i}$ is either + or - ,

- oriented ribbon tangles as morphisms, colored by morphisms of $\mathscr{X}$, and considered modulo topological deformation.

So, a typical morphism

$$
\left(A^{+}\right) \quad \longrightarrow \quad\left(B^{+}, C^{-}, D^{+}\right)
$$

in the category free-ribbon $(\mathscr{X})$ looks like this

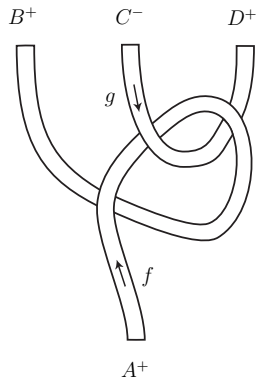

where $f: A \longrightarrow B$ and $g: C \longrightarrow D$ are morphisms in the category $\mathscr{X}$. Now, consider the full and faithful functor

\section{$\mathscr{X} \longrightarrow$ free-ribbon $(\mathscr{X})$}

which transports every object $A$ of the category $\mathscr{X}$ to the signed sequence $\left(A^{+}\right)$in the category free-ribbon $(\mathscr{X})$. Then, just as in the case of the free cartesian closed category, every functor from the category $\mathscr{X}$ to a ribbon category $\mathscr{D}$ extends to a structure-preserving functor [-] which makes the diagram below commute:

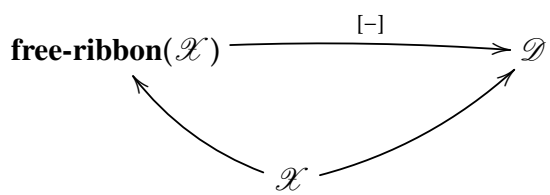

The point is that, once properly oriented and colored, every topological knot $P$ defines a morphism $P: I \longrightarrow I$ from the tensorial unit $I=()$ to itself in the category free-ribbon $(\mathscr{X})$. From this follows that the image $[P]$ defines a invariant of the knot $P$ modulo topological deformation. Among other applications, the method enables one to establish that the Jones polynomial $[P]$ associated to a knot $P$ defines indeed a topological invariant, see [13] for details.

Dialogue categories. We have just pointed out a similarity between the conceptual tools developed in order to study topological knots and logical proofs. A key difference however is that the definition of free-ccc $(\mathscr{X})$ is based on a symbolic calculus, the simply-typed $\lambda$-calculus, instead of a topological notation, as in the definition of free-ribbon $(\mathscr{X})$. At this point, it is worth asking oneself whether the notion of cartesian closed category could be fine-tuned and replaced by a notion closer to ribbon categories... whose stringy nature could be then uncovered.

It appears that this question leads again to tensorial logic - and more specifically to the corresponding notion of dialogue category. A dialogue category is defined as a symmetric monoidal category $\mathscr{D}$ equipped with an exponentiating object $\perp$. Recall that

Definition 1 (exponentiating object): An exponentiating object in a symmetric monoidal category $C$ is an object $\perp$ equipped for every object $a$ with an object $a \multimap \perp$ and with a bijection

$$
\mathscr{D}(a \otimes b, \perp) \cong \mathscr{D}(b, a \multimap \perp)
$$

natural in the object $b$.

Definition 2 (dialogue categories): A dialogue category is a symmetric monoidal category equipped with an exponentiating object.

Hence, the notion of dialogue category reflects tensorial logic and its two logical connectives:

- the linear conjunction $\otimes$ and its unit $I$,

- the linear negation $a \mapsto \neg a$ defined as $a \multimap \perp$

in exactly the same way as the notion of cartesian closed category reflects (minimal) intuitionistic logic. A typical example of dialogue category is provided by the category of vector spaces (of finite and infinite dimension) on a base field $k$, with $\perp=k$. In that case, the negation of a vector space $V$ is its dual vector space $V^{*}$ of linear forms from $V$ to $k$. Another example of dialogue category is provided by the category Set of sets and functions equipped with a set $\perp$ chosen as return type. More generally, any symmetric monoidal closed category $\mathscr{D}$ equipped with an arbitrary object $\perp$ as return type. Another important class of example is provided by $*$-autonomous categories with the dualizing object $\perp$ as return type. Note that a*autonomous category may be seen as a degenerate dialogue category where the canonical map $a \longrightarrow \neg \neg a$ is invertible.

Dialogue games. The notion of innocent strategy introduced by Hyland and Ong in dialogue games [11] provides us with a clean abstract and combinatorial notion of proof (and program) which may be formulated in three different styles, with clear transcriptions between them:

- the traditional syntax of $\lambda$-terms, because an innocent strategy is generated by a (possibly infinite) Bohm tree, whose branches describe the so-called Proponent views of the innocent strategy,

- the dynamic semantics provided by game semantics, because an innocent strategy is a particular kind of interactive strategy which plays according to its current view of the play, rather than according to the play itself,

- the static semantics provided by relational semantics, because an innocent strategy is positional in asynchronous games [19] and thus entirely described by its set of halting positions.

One main observation of our work is that game semantics should be understood as a syntax of tensorial proofs, expressed at the same time 
graphically as a flow of control. As a matter of fact, we show how to apply the algebraic principles of knot theory to this flow of control, and to characterize the free dialogue category free-dialogue $(\mathscr{X})$ generated by a category $\mathscr{X}$ in the same way as one characterizes the free ribbon category free-ribbon $(\mathscr{X})$.

Namely, we will exhibit a series of basic logical principles of tensorial logic (or generating proofs) providing a logical counterpart to the topological generators of framed tangles (braidings and Uturns) appearing in the construction of the free ribbon category free-ribbon $(\mathscr{X})$. Using this decomposition of tensorial proofs in basic blocks, we establish that the category free-dialogue $(\mathscr{X})$ has

- dialogue games as objects, with objects of the category $\mathscr{X}$ considered as atomic games,

- total innocent strategies as morphisms, where the terminal moves between atomic games are colored by a morphism of $\mathscr{X}$.

We believe that this coherence theorem for dialogue category provides a firm algebraic foundation to the traditional "full completeness theorems" of game semantics obtained in [1], [11] and to the connection between innocent strategies and linear continuations stressed in [23], [24]. This result also demonstrates that game semantics is a syntax as much as a semantics, since every functor $\mathscr{X} \longrightarrow \mathscr{D}$ to a dialogue category induces a structure-preserving functor [-] which makes the diagram below commute:

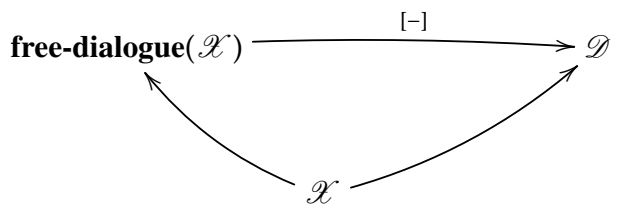

In particular, the coherence theorem establishes that every dialogue category $\mathscr{D}$ provides a semantic interpretation of innocent strategies. A typical illustration of this phenomenon is provided by the dialogue category $\mathscr{D}$ of vector spaces (of a finite or infinite dimension) on a field $k$, which provides a denotational semantics of tensorial logic, where every innocent strategy $\sigma$ of a dialogue game $A$ is interpreted as a vector $[\sigma]$ in the appropriate vector space $[A]$. This shows that game theoretical structures appear in somewhat unexpected fields of mathematics.

Similarly, the category $\mathscr{D}$ of coherence space with $\perp=1$ is a $*$ autonomous category and thus a dialogue category: the coherence theorem enables us to extract the static content of an innocent strategy $\sigma$ of the dialogue game $A$ by transporting it to a clique $[\sigma]$ in the appropriate coherence space $[A]$.

Plan of the paper. We recall the notion of innocent strategy in dialogue games in $\S$ II. We then study in $\S$ III the logic of pure negation (that is, tensorial logic without its tensor product) and establish that it is generated by the adjunction of negation with itself. This uncovers the unit $\eta$ and counit $\varepsilon$ of this self-adjunction as our two first generators of tensorial proof-nets. Once dialogue categories have been reformulated as dialogue chiralities in $\S \mathrm{IV}$, we describe in $\S \mathrm{V}$ a pair of distributivity laws $\kappa_{\emptyset}$ and $\kappa_{\otimes}$, alternatively seen as parametric strengths, which provide the second pair of generators of tensorial proof-nets. We conclude our algebraic presentation in $\S$ VI with two generators axiom and cut reflecting the axioms and cuts of tensorial logic. We then establish in $\S$ VIII our main technical result: a factorization theorem, which states that every sequence of generators factors as a sequence of Opponent combinators: $\varepsilon, \kappa_{\mathbb{Q}}$, cut followed by a sequence of Proponent combinators: $\eta, \kappa_{\emptyset}$, axiom, providing a purely combinatorial account of innocence. The coherence theorem is then deduced from this factorization property in $\S$ IX. We describe the related works in $\S \mathrm{X}$.

\section{Dialogue Games And Innocent StRategies}

In this section, we define the category of dialogue games and innocent strategies generated by a category $\mathscr{X}$ of atoms. The definition follows the pattern of [19] except for the careful treatment of the atomic moves reflecting the objects and morphisms of the underlying category $\mathscr{X}$.

Dialogue games. A dialogue game is a quadruple

$$
A=\left(M_{A}, \leq_{A}, \lambda_{A}, o_{A}\right)
$$

consisting of:

- a finite forest $\left(M_{A}, \leq_{A}\right)$ whose nodes are called the moves of the game,

- a function $\lambda_{A}: M_{A} \longrightarrow\{-1,+1\}$ which associates to every move a polarity +1 (for the Player moves) or -1 (for the Opponent moves),

- a function $o_{A}: M_{A} \longrightarrow \mathbf{o b j}(\mathscr{X})+\{\bullet\}$ which associates to every move a label consisting of an object of the category $\mathscr{X}$ or the tag $\bullet$.

A move labeled by $\bullet$ is called a regular move, whereas a move labeled by an object of $\mathscr{X}$ is called an atomic move. Three conditions are moreover required on a dialogue game:

- the nodes at the root of the forest are required to be Opponent moves,

- the forest is required to be bipartite: the polarities of nodes are alternating on every branch,

- the atomic moves are required to be at the leaves of the forest. Note that the converse of the third condition does not hold: a move at the leaves of the forest is not required to be atomic. We say that a move $m$ justifies another move $n$ of an arena game $A$ and write $m \vdash_{A} n$ when $m$ is the immediate predecessor of $n$, that is, $m \leq_{A} n$ and for every move $p$ of the arena game,

$$
m \leq_{A} p \leq_{A} n \Rightarrow m \leq_{A} p \leq_{A} n \Rightarrow m=p \text { or } p=n .
$$

Positions. A position of the game is a downward closed set of nodes of the forest. Every position may be thus seen as a partial exploration of the forest, starting from the roots.

The asynchronous graph. Every dialogue game $A$ induces a graph $\mathscr{G}_{A}$ :

- its nodes are the positions $x, y \in \mathscr{D}_{A}$,

- its edges $m: x \longrightarrow y$ are the moves verifying $y=x \uplus\{m\}$, where $\uplus$ denotes disjoint union, or equivalently, that $y=x \cup\{m\}$ and that the move $m$ is not an element of $x$.

This graph $\mathscr{G}_{A}$ is called the asynchronous graph of the dialogue game $A$. We write $s: x \longrightarrow y$ for a path

$$
x \stackrel{m_{1}}{\rightarrow} x_{1} \stackrel{m_{2}}{\rightarrow} \cdots \stackrel{m_{k-1}}{\rightarrow} x_{k-1} \stackrel{m_{k}}{\rightarrow} y
$$

between two positions $x$ and $y$.

Alternating paths. A path $m_{1} \cdots m_{k}: x \longrightarrow y$ is alternating when:

$$
\forall i \in\{1, \ldots, k-1\}, \quad \lambda_{A}\left(m_{i+1}\right)=-\lambda_{A}\left(m_{i}\right) .
$$

Legal plays. A legal play is a pair $(s, \varphi)$ consisting of an alternating path $s$ starting from the empty position $*_{A}$ in the asynchronous graph $\mathscr{G}_{A}$ :

$$
*_{A} \stackrel{m_{1}}{\rightarrow} x_{1} \stackrel{m_{2}}{\rightarrow} \cdots \stackrel{m_{2 k-1}}{\rightarrow} x_{2 k-1} \stackrel{m_{2 k}}{\rightarrow} x_{2 k}
$$

together with a word of length $k$ consisting of the letter $\bullet$ or a morphism of the category $\mathscr{X}$, such that, for all $0 \leq i \leq k-1$ :

$$
\begin{gathered}
\varphi(i)=\bullet \Rightarrow o\left(m_{2 i-1}\right)=\bullet \text { and } o\left(m_{2 i}\right)=\bullet \\
\varphi(i)=f: A \longrightarrow B \quad \Rightarrow \quad o\left(m_{2 i-1}\right)=B \text { and } o\left(m_{2 i}\right)=A .
\end{gathered}
$$


In that last case, we find useful to write the morphism $f$ as a double pointer from the move $m_{2 i}$ to the move $m_{2 i-1}$, as follows:

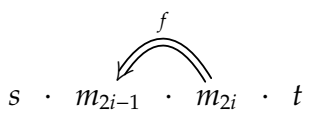

It should be stressed that the double pointer does not mean that the move $m_{2 i}$ is justified by the move $m_{2 i-1}$. This is never the case in fact, since an atomic move like $m_{2 i-1}$ is a leaf of the dialogue game, and thus does not justify any other move. The set of legal plays is noted $P_{A}$.

Strategy. A strategy $\sigma$ is a set of legal plays such that:

- the strategy $\sigma$ contains the empty play,

- $\sigma$ is closed by even-length prefix:

$$
\forall s \in P_{A}, \forall m, n \in M_{A}, \quad s \cdot m \cdot n \in \sigma \Rightarrow s \in \sigma,
$$

- $\sigma$ is deterministic: $\forall s \in P_{A}, \forall m, n_{1}, n_{2} \in M_{A}$,

$$
s \cdot m \cdot n_{1} \in \sigma \text { and } s \cdot m \cdot n_{2} \in \sigma \Rightarrow n_{1}=n_{2} .
$$

One should be careful here with the fact that a play is defined as a path $s$ equipped with an additional structure $\varphi$. In particular, determinism means that whenever the two plays

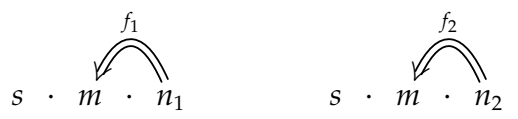

are element of the strategy $\sigma$, then the two moves $n_{1}$ and $n_{2}$ coincide, as well as the two morphisms $f_{1}$ and $f_{2}$ of the category $\mathscr{X}$.

View extraction. The binary relation $\stackrel{\mathrm{OP}}{\rightsquigarrow}$ is defined as the smallest relation between alternating plays such that:

$$
s_{1} \cdot m \cdot n \cdot s_{2} \quad \stackrel{\text { OP }}{\rightsquigarrow} s_{1} \cdot s_{2}
$$

for every alternating play $s_{1}$ of even-length, every nonempty alternating path $s_{2}$, every Opponent move $m$ which does not justify any move in $s_{2}$, and every Player move $n$ which does not justify any move in $s_{2}$.

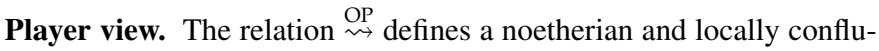
ent rewriting system on alternating plays. By Newman's Lemma, the rewriting system is confluent. Thus, every alternating play $s$ induces a unique normal form noted $\lceil s\rceil$ and called the Player view of $s$ :

$$
s \stackrel{\mathrm{OP}}{\rightsquigarrow} s_{1} \stackrel{\mathrm{OP}}{\rightsquigarrow} \cdots \stackrel{\text { OP }}{\rightsquigarrow} s_{k} \stackrel{\text { OP }}{\rightsquigarrow}\lceil s\rceil .
$$

Innocent strategies. A strategy $\sigma$ is called innocent in a dialogue game $A$ when for all plays $s, t \in \sigma$, for every Opponent move $m \in M_{A}$ and Player move $n \in M_{A}$, the following situation

$$
s \cdot m \cdot n \in \sigma \text { and } t \cdot m \in P_{A} \text { and }\lceil s \cdot m\rceil=\lceil t \cdot m\rceil
$$

implies that

$$
t \cdot m \cdot n \in \sigma
$$

Once again, we should be careful about the additional structure $\varphi$ equipping the path $s$. In particular, innocence means that the two legal plays

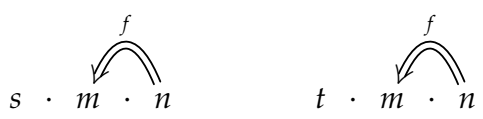

in the strategy $\sigma$ are labeled by the same morphism

$$
f: o(n) \longrightarrow o(m)
$$

in the underlying category $\mathscr{X}$.
Remark. The careful reader will notice that the morphisms in the category $\mathscr{X}$ go in the reverse direction as the flow of control in the play. Typically, we will see very soon that the strategy associated to the cut link of tensorial logic plays the moves in this order:

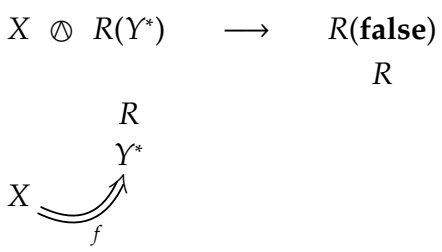

where the morphism $f: A \longrightarrow B$ of the category $\mathscr{X}$ is indicated with a pointer from the atomic move $A$ to the atomic move $B$.

Total strategies. A strategy is total when every maximal play in the strategy contains all the moves of the underlying dialogue game $A$. Note that this notion is stronger than the usual notion of totality, since it requires that all the moves of the arena game $A$ are eventually played by the strategy.

Tensor product. The tensor product of two dialogue games $A$ and $B$ is defined as the dialogue game

$$
M_{A \otimes B}=M_{A}+M_{B}, \quad \lambda_{A \otimes B}=\left[\lambda_{A}, \lambda_{B}\right], \quad o_{A \otimes B}=\left[o_{A}, o_{B}\right] .
$$

Negation. The negation of a dialogue game $A$ is defined as the dialogue game $\neg A$ where the polarities of moves have been reversed, lifted by a new initial move $m$ played by Opponent:

$$
M_{(\neg A)}=M_{A}+\{m\}, \quad \lambda_{(\neg A)}=-\lambda_{A}, \quad o_{(\neg A)}=o_{A}
$$

together with $\lambda_{(\neg A)}(m)=-1$ and $o_{(\neg A)}(m)=\bullet$.

The category of dialogue games. The category of dialogue games generated by the category $\mathscr{X}$ is denoted innocent $(\mathscr{X})$ and defined as follows:

- its objects are the dialogue games with atoms in $\mathscr{X}$,

- its morphisms $A \longrightarrow B$ are the total innocent strategies of the game $A \multimap B$.

Here, the game $A \multimap B$ is defined as the game

$$
M_{A \multimap B}=M_{A}+M_{B}, \quad \lambda_{A \multimap B}=\left[-\lambda_{A}, \lambda_{B}\right], \quad o_{A \multimap B}=\left[o_{A}, o_{B}\right] .
$$

Note in particular that the the polarities of the moves in the game $A$ have been reversed. From this follows that the game $A \multimap B$ is not exactly a dialogue game, since the initial moves in $A$ are Player moves, instead of Opponent moves. However, an innocent strategy $\sigma$ in the game $A \multimap B$ may be defined as an innocent strategy in the underlying asynchronous graph $\mathscr{G}_{A \multimap B}$, where we are careful to ask that the legal plays in $\mathscr{G}_{A \multimap B}$ start by an Opponent move (thus in the component $B$ ).

Now, let $\perp$ denote the dialogue game with a unique Opponent move labelled $\bullet$. Note that the morphisms from any dialogue game $A$ to $\perp$ in the category innocent $(\mathscr{X})$ are the same as the innocent strategies in the dialogue game $\neg A$. From this follows that:

Proposition 3: The category innocent $(\mathscr{X})$ equipped with the singleton game $\perp$ defines a dialogue category. Moreover, there is a functor

$$
\iota: \mathscr{X} \longrightarrow \operatorname{innocent}(\mathscr{X})
$$

which transports

- every object $X$ to the dialogue game $\iota X$ consisting of a unique Opponent move labeled by the object $X$, 
- every morphism $f: X \longrightarrow Y$ to the strategy

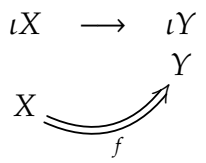

One main purpose of the following sections is to establish that the category $\operatorname{innocent}(\mathscr{X})$ coincides with the free dialogue category generated by the category $\mathscr{X}$.

\section{A SELF-AdJUNCTION}

A first observation is that every exponentiating object $\perp$ in a symmetric monoidal category induces a pair of functors

$$
L: \mathscr{C} \longrightarrow \mathscr{C}^{o p} \quad R: \mathscr{C}^{o p} \longrightarrow \mathscr{C}
$$

transporting an object $a$ into its negation $a \multimap \perp$ in the opposite category. The series of natural bijections

$$
\begin{aligned}
\mathscr{C}(a, R(b)) & \cong \mathscr{C}(a, b \multimap \perp) \\
& \cong \mathscr{C}(a \otimes b, \perp) \\
& \cong \mathscr{C}(b, a \multimap \perp) \cong \mathscr{C}^{o p}(L(a), b)
\end{aligned}
$$

establishes moreover that the functor $L$ is left adjoint to the functor $R$. The starting point of our work is the observation that the adjunction $L \dashv R$ of negation with itself plays in proof theory the same role as duality in knot theory. In order to establish this correspondence, we need to shift from string diagrams in monoidal categories to string diagrams in 2-categories, taking advantage of Street's algebraic presentation of adjunctions $L \dashv R$ performed in the language of 2-categories [28], see also [20]. At this point, we refer to Selinger's nice introduction to string diagrams [27] and more specifically his Section 8 explaining the specific case of 2-categorical string diagrams.

Now, recall that every adjunction $L \dashv R$ induces a monad $R \circ L$ and a comonad $L \circ R$. In Street's presentation, the two generators of the adjunction $L \dashv R$ are the unit $\eta$ of the $\operatorname{monad} R \circ L$ and the counit $\varepsilon$ of the comonad $L \circ R$ :

$$
\varepsilon: L \circ R(b) \longrightarrow b \quad \eta: a \longrightarrow R \circ L(a)
$$

These two generators of the adjunction $L \dashv R$ are nicely depicted in the language of string diagrams for 2-categories:
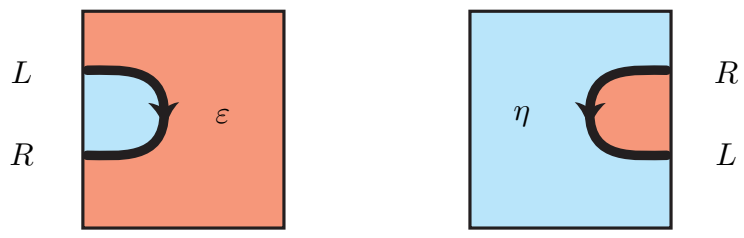

The main novelty of these string diagrams compared to the familiar string diagrams of monoidal categories is that they are multi-coloured:

- the blue surface represents the category $\mathscr{C}$,

- the red surface represents the opposite category $\mathscr{C}^{o p}$,

- the negation functors $R$ and $L$ are depicted as oriented strings separating a blue surface from a red surface.

So, a typical proof from an atomic formula $A$ negated six times to the same atomic formula $A$ negated four times looks like this:

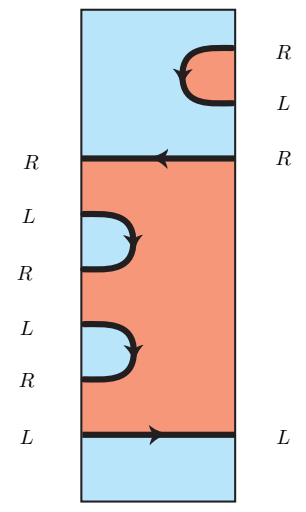

The correspondence with game semantics is striking at this point: if one reads the diagram topdown, and understands the orientation of the negation strands as the intended flow of control, then it appears that the flow of negations $R$ and $L$ described by the string diagram implements an interactive strategy playing the negation functors $R$ as input tokens (or Opponent moves) and the negation functors $L$ as output tokens (or Proponent moves). The fact that each functor $R$ and $L$ shifts from the category $\mathscr{C}$ to the category $\mathscr{C}^{o p}$ and conversely captures the basic intuition that a negation is a turn in the dialogue game, where control is exchanged between players.

Composition of proofs is then performed by gluing the diagrams in the expected way:

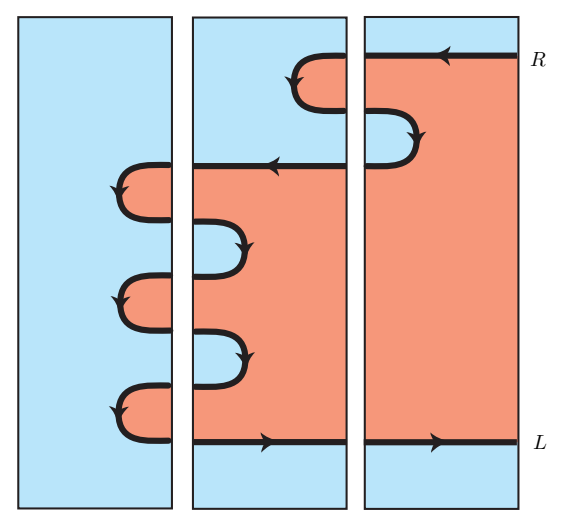

The evaluation mechanism of game semantics (sometimes called interaction plus hiding) is then implemented by the pair of triangular laws regulating the generators $\eta$ and $\varepsilon$ in the algebraic presentation of adjunctions, see [28]. Diagrammatically speaking, these relations are the counterpart of the "zig-zag" relation for dual pairs, and thus describe the topological deformation of a curve into a straight line:
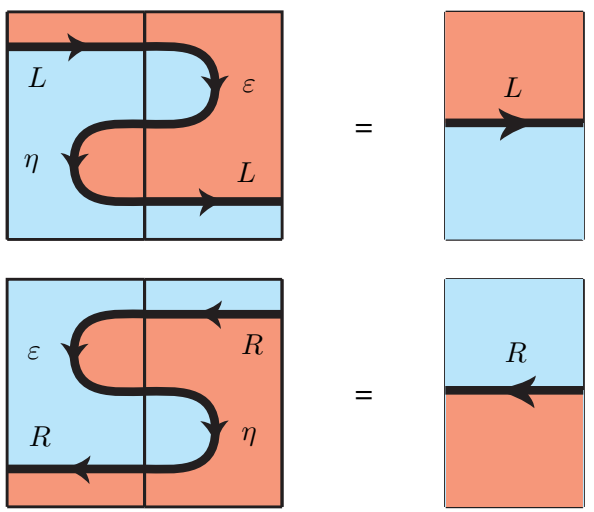

These two relations imply in particular that the composite proof depicted above is equal to the generator $\eta$. An important observation is that all the proofs of this pure logic of negation are generated by 
the unit $\eta$ and the counit $\varepsilon$ of the adjunction $L \dashv R$. This means that this most elementary fragment of logic is entirely described by the algebraic fact that negation is adjoint to itself.

\section{Dialogue Categories as Dialogue Chiralities}

Every monoidal category $\mathscr{C}=(\mathscr{C}, \otimes, I)$ induces a monoidal category noted

$$
\mathscr{C}^{o p(0,1)}=\left(\mathscr{C}^{o p(1)}, \otimes^{o p(0)}, I\right)
$$

with the opposite category $\mathscr{C}^{o p(1)}=\mathscr{C}^{o p}$ as underlying category, and with the tensor product $\otimes^{o p(0)}$ defined by permuting the two components of the original tensor product:

$$
a \otimes^{o p(0)} b=b \otimes a .
$$

Dialogue categories are governed by a chirality principle which says that every map in a dialogue category $\mathscr{C}$ may be seen at the same time as a map in the opposite category $\mathscr{C}^{o p(0,1)}$. This idea leads to the following notion of refraction chirality which enables to manipulate the two categories $\mathscr{C}$ and $\mathscr{C}^{o p(0,1)}$ on a symmetric and equal footing, see [?] for details. A refraction chirality is defined as a pair of symmetric monoidal categories

$$
(\mathscr{A}, \otimes, \text { true }) \quad(\mathscr{B}, \otimes, \text { false })
$$

equipped with a symmetric monoidal equivalence

$$
(-)^{*}: \mathscr{A} \longrightarrow \mathscr{B}^{o p(0,1)} \quad: \quad{ }^{*}(-)
$$

a distributor, or categorical bimodule

$$
\langle-\mid-\rangle: \mathscr{A}^{o p} \times \mathscr{B} \quad \longrightarrow \text { Set }
$$

and a family of bijections

$$
\chi_{m, a, b}:\langle a \oplus m \mid b\rangle \longrightarrow\left\langle a \mid b \otimes m^{*}\right\rangle
$$

natural in $a$ and $b$, making the diagram below commute:

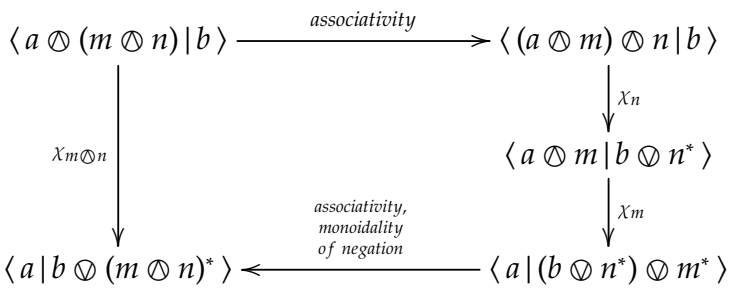

A dialogue chirality is then defined as a refraction chirality whose distributor comes from an adjunction $L \dashv R$ in the sense that

$$
\langle a \mid b\rangle=\mathscr{A}(a, R(b)) .
$$

Note that every dialogue category $\mathscr{C}$ induces a dialogue chirality $G(\mathscr{C})$ where

$$
\mathscr{A}=\mathscr{C} \quad \mathscr{B}=\mathscr{C}^{o p(0,1)}
$$

and the two complementary functors $(-)^{*}$ and ${ }^{*}(-)$ are the identity functor on the monoidal category $\mathscr{C}$, and the adjunction $L \dashv R$ is deduced from the negation as in Section III. At this point, it is not extremely difficult (although it should be done with care) to construct a suitable 2-category DiaCat of dialogue categories and a suitable 2-category DiaChir of dialogue chiralities, in such a way that this construction $\mathcal{G}$ defines a 2-dimensional equivalence between DiaCat and DiaChir. In particular, every dialogue chirality is equivalent in the 2-category DiaChir to the image $\mathcal{G}(\mathscr{C})$ of a dialogue category $\mathscr{C}$. In other words, every dialogue chirality may be "strictified" into a dialogue category, this fact establishing the notion of dialogue chirality as a relaxed but equivalent notion of dialogue category. So, we will generally confuse the two notions in the sequel, and think of a dialogue chirality as a properly symmetric way to formulate a dialogue category.

\section{A Chiral Pair of Distributivity LaWs}

Reformulating dialogue categories as dialogue chiralities enables one to apply the chirality principle, which states that every generator of tensorial logic in the category $\mathscr{A}=\mathscr{C}$ has a "mirror picture" in the category $\mathscr{B}=\mathscr{C}^{o p}$, defining a dual generator. This chirality principle is nicely illustrated by the fact that the generator $\varepsilon$ is the mirror picture in the category $\mathscr{B}$ of the generator $\eta$ in the category $\mathscr{A}$. In the same way, the chirality principle enables one to "replicate" the distributivity law (3) mentioned in the introduction

$$
a_{1} \otimes \neg\left(b \otimes \neg a_{2}\right) \longrightarrow \neg\left(b \otimes \neg\left(a_{1} \otimes a_{2}\right)\right)
$$

as a dual pair $\left(\kappa_{\otimes}, \kappa_{\otimes}\right)$ of distributivity laws, consisting of a "conjunctive" distributivity law $\kappa_{\bigotimes}$ living in the category $\mathscr{A}$ and of a "disjunctive" distributivity law $\kappa_{\emptyset}$ living in the category $\mathscr{B}$

$$
\begin{array}{lll}
a_{1} \otimes R\left(L\left(a_{2}\right) \otimes b\right) & \stackrel{\kappa_{\otimes}\left(a_{1}, a_{2}, b\right)}{\longrightarrow} & R\left(L\left(a_{1} \otimes a_{2}\right) \otimes b\right) \\
L\left(R\left(b_{1} \otimes b_{2}\right) \otimes a\right) & \stackrel{\kappa_{\circledast}\left(b_{1}, b_{2}, a\right)}{\longrightarrow} & b_{1} \otimes L\left(R\left(b_{2}\right) \otimes a\right)
\end{array}
$$

It is worth observing that:

- the conjunctive distributivity law $\kappa_{\otimes}$ imports $a_{1} \otimes-$ inside the scope of the two negations $R$ and $L$,

- the disjunctive distributivity law $\kappa_{\emptyset}$ exports $b_{1} \oslash$ - outside the scope of the two negations $R$ and $L$.

On the other hand, the two distributivity laws $\kappa_{\otimes}$ and $\kappa_{\emptyset}$ import a conjunction $\otimes$ inside a disjunction $\otimes$, thus explaining why both laws are identified when one collapses tensorial logic to linear logic by "removing" the negations.

Just as in the case of ribbon knots in ribbon categories, the 2-dimensional string diagrams of linear negation (in Section III) describe particles in motion. In order to interpret the tensor product of tensorial logic, one needs to consider surface diagrams describing formula trees (rather than simply particles) in motion. These surface diagrams are performed in the traditional notation for monoidal (or in that case cartesian) 2-categories, see for instance Street [29]. By way of illustration, consider the sequent

$$
\neg \neg a \otimes \neg \neg b \quad \vdash \quad \neg \neg(a \otimes b)
$$

alternatively written as

$$
R L(a) \oplus R L(b) \quad \vdash \quad R L(a \oplus b)
$$

in the style of dialogue chiralities. The left-to-right proof of the sequent factors then as a composite of three basic combinators:

$$
\begin{array}{rlc}
R L(a) \oplus R L(b) \quad \stackrel{{ }_{\oplus}}{\longrightarrow} & R L(a \oplus R L(b)) \\
& \stackrel{\kappa_{\oplus}}{\longrightarrow} & R L R L(a \oplus b) \\
& \stackrel{R L(a \oplus b)}{\longrightarrow} & R
\end{array}
$$

Accordingly, the proof is depicted in string diagrams as the composite of the three combinators, describing a formula tree in motion:

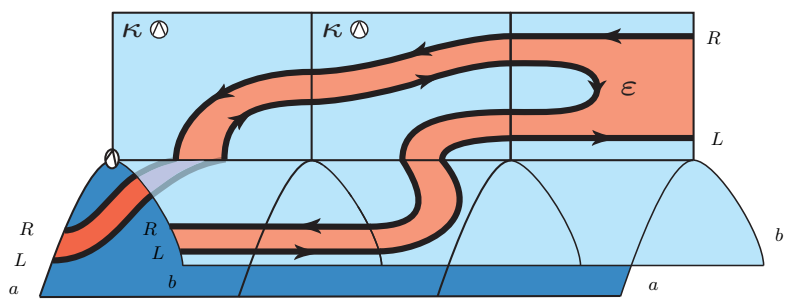


Note that the very same proof of tensorial logic may be formulated in the sequent calculus:

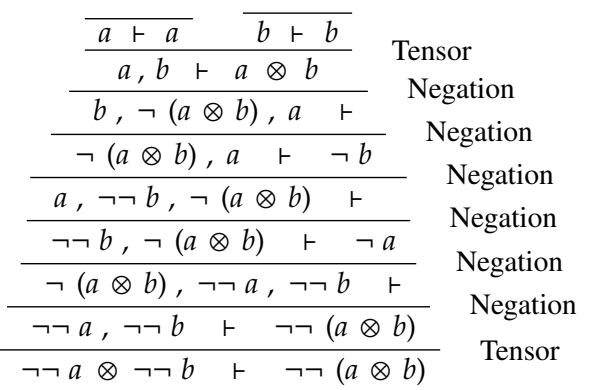

The flow of negations appearing in the string diagram coincides with the game-theoretic interpretation of the derivation tree as an innocent strategy in dialogue games:

\begin{tabular}{|c|c|c|c|c|c|}
\hline & $R L(a)$ & (1) & $R L(b)$ & r & $R L(a \oplus b)$ \\
\hline move 1. & & & & & $R$ \\
\hline move 2 . & $R$ & & & & \\
\hline move 3 . & $L$ & & & & \\
\hline move 4 . & & & $R$ & & \\
\hline move 5. & & & $L$ & & \\
\hline move 6. & & & & & $L$ \\
\hline
\end{tabular}

\section{AxIOMS AND CUTS}

At this point, we describe the two generators cut and axiom corresponding to atomic axiom and cut rules in tensorial logic. Every morphism

$$
f: X \rightarrow Y
$$

of the category $\mathscr{X}$ induces a pair of generators

$$
\begin{array}{ccc}
X \otimes R\left(Y^{*} \otimes b\right) & \frac{{ }^{c u t_{f, b}}}{\longrightarrow} & R b \\
L a & \stackrel{{ }^{a x_{f, a}} \longrightarrow}{\longrightarrow} & X^{*} \otimes L(Y \otimes a)
\end{array}
$$

which are depicted in the language of string diagrams as
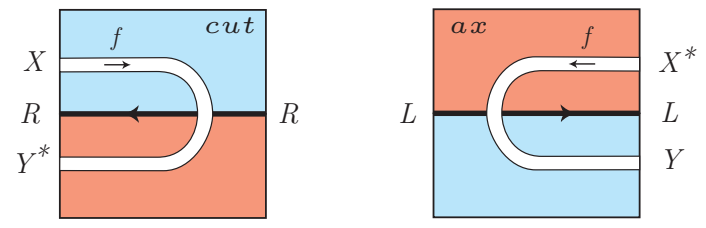

Here, the white wire $X$ on the category $\mathscr{A}$ (blue background) depicts the functor

$$
a \mapsto X \otimes a: \mathscr{A} \longrightarrow \mathscr{A}
$$

whereas the white wire $Y$ on the category $\mathscr{B}$ (red background) depicts the functor

$$
b \mapsto Y \otimes b \quad: \quad \mathscr{B} \quad \longrightarrow \quad \mathscr{B} .
$$

Accordingly, given a morphism (6) in the underlying category $\mathscr{X}$, the arrow $f$ tagged on the white wire in $\mathscr{A}$ (blue background) depicts the natural transformation

$$
(X \otimes-) \Rightarrow(Y \otimes-): \quad \mathscr{A} \longrightarrow \mathscr{A}
$$

whereas the arrow $f$ tagged on the white wire in $\mathscr{B}$ (red background) depicts the natural transformation

$$
\left(Y^{*} \otimes-\right) \Rightarrow\left(X^{*} \oslash-\right): \mathscr{B} \longrightarrow \mathscr{B} .
$$

Note that the two generators cut and axiom are the chiral transcriptions of the sequent

$$
X \otimes \neg(A \otimes Y) \quad \vdash \quad \neg A
$$

which is provable in tensorial logic by the derivation tree

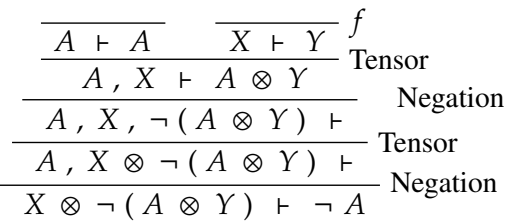

The interaction between the generators axiom and cut is regulated by two relations for each pair of morphisms $f: X \longrightarrow Y$ and $g: Y \longrightarrow Z$ in the category $\mathscr{X}$ :

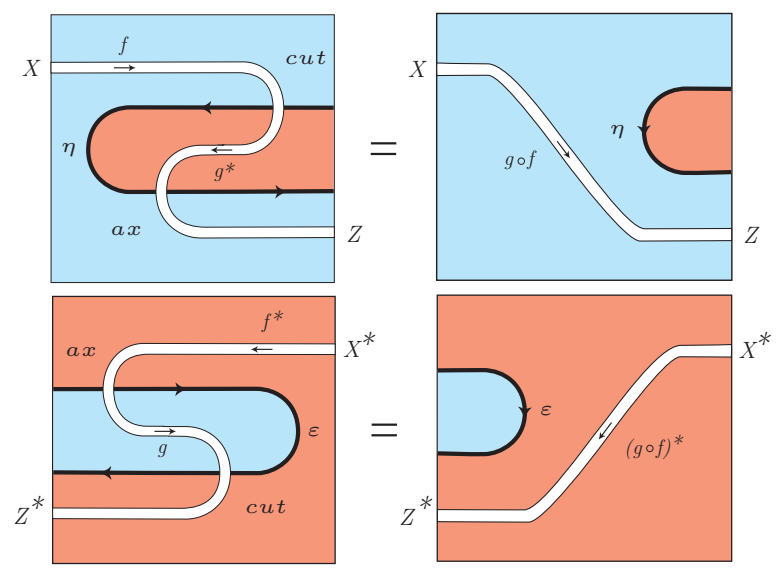

The generators axiom and cut together with the two relations above express that there exists a family of adjunctions

$$
L(X \otimes-) \quad \dashv \quad R\left(X^{*} \otimes-\right)
$$

natural in the object $X$. Note that the equality reduces to the cutelimination rule of proof-nets in linear logic in the degenerate case when $L=R=i d$.

\section{THE MAPS}

We describe the very last two generators of our algebra presentation of the free dialogue category free-dialogue $(\mathscr{X})$ on a category $\mathscr{X}$. The idea is that every morphism

$$
f: X \rightarrow Y
$$

of the original category $\mathscr{X}$ induces a pair of generators:

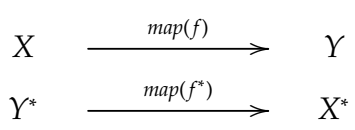

depicted as follows in our string diagrams:
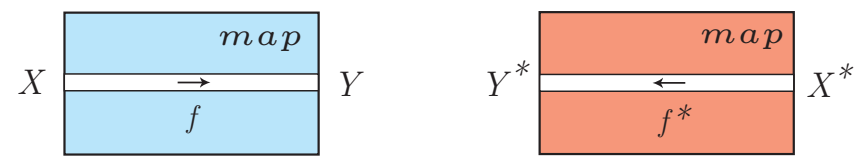

\section{The OpPonent-Proponent FACTORIZATION TheOREM}

In order to prove our coherence theorem for dialogue categories in Section IX, we establish that

Theorem 4 (Factorization): Every sequence of generators picked among

$$
\varepsilon \quad \eta \quad \kappa_{\otimes} \quad \kappa_{\circledast} \quad \text { cut axiom map }
$$

transforming a formula $F$ into a formula $G$ may be reorganized as a sequence of "Opponent" generators (or combinators)

$$
\varepsilon \quad \kappa_{\otimes} \quad \text { cut }
$$


transforming the formula $F$ into a formula $H_{1}$, followed by a sequence of generators

\section{map}

transforming the formula $H_{1}$ into a formula $H_{2}$, followed by a sequence of "Proponent" generators (or combinators)

$$
\eta \quad \kappa_{\circledast} \text { axiom }
$$

transforming the formula $\mathrm{H}_{2}$ into the formula $G$.

The reorganization is achieved thanks to a family of basic rewrite rules $\Rightarrow$ which may be seen as the resolution of a critical pair in another term rewriting system $\mathcal{R}$ on tensorial formulas, whose rewriting rules are:

- the Opponent generators $\varepsilon, \kappa_{\otimes}$ and cut,

- the Proponent generators $\eta, \kappa_{\circledast}$ and axiom, taken in the reverse direction: $\bar{\eta}, \overline{\kappa_{\oslash}}$ and $\overline{\text { axiom }}$,

- the map generator map.

By way of illustration, one of these rewriting rules orients the coherence law of the monadic strength, which appears in this way as a local confluence diagram for the critical pair between the two rewriting rules $\bar{\eta}: a_{1} \otimes R L\left(a_{2}\right) \rightarrow a_{1} \otimes a_{2}$ and $\kappa_{\otimes}: a_{1} \otimes R L\left(a_{2}\right) \rightarrow R L\left(a_{1} \otimes a_{2}\right):$

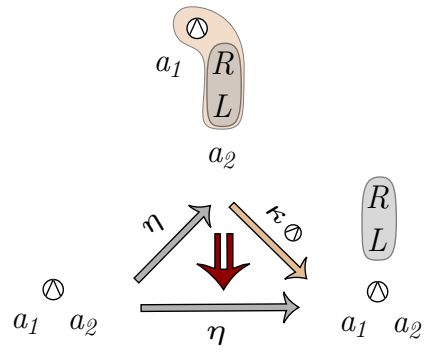

The overlapping patterns of the critical pair in the rewriting rules in $\mathcal{R}$ are colored in the term $a_{1} \otimes R L\left(a_{2}\right)$. The key observation is that the rewriting system $\mathcal{R}$ is strongly normalizing, at least when one removes the rewriting rule map, and restricts it to the non trivial instances of the rewriting rules $\kappa_{\bigotimes}$ and $\kappa_{\otimes}$. From this follows that the rewriting system $\Rightarrow$ itself is strongly normalizing, and thus reaches a normal form, which is a properly factorized sequence of transformations from $F$ to $G$. This establishes our claim. To this, one should add the fact that all the rewriting rules $\Rightarrow$ between paths are commutative in a dialogue category - this point ensuring that the factorization procedure preserves the meaning of the morphism in any dialogue category.

\section{The COHERENCE THeOREM}

Once the Opponent-Proponent factorization theorem is established for the proof $\pi: F \vdash G$, one may focus on the resulting sequence of "Opponent" generators from the formula $F$ to the formula $H_{1}$, and show by a simple combinatorial argument that every such sequence of generators factors as

$$
F \stackrel{\kappa \otimes}{\longrightarrow} J_{1} \stackrel{\text { cut }}{\longrightarrow} J_{2} \stackrel{\varepsilon}{\longrightarrow} H_{1}
$$

where each arrow is labeled by the name $\kappa_{\mathbb{Q}}$, cut and $\varepsilon$ of the generators appearing in it. Symmetrically, one establishes that the sequence of "Proponent" generators from $\mathrm{H}_{2}$ to $\mathrm{G}$ factors as

$$
H_{2} \stackrel{\eta}{\longrightarrow} K_{2} \stackrel{\text { axiom }}{\longrightarrow} K_{1} \stackrel{\kappa_{\circledast}}{\longrightarrow} G
$$

This decomposition of the proof $\pi: F \vdash G$ provides an algebraic counterpart to the notion of innocent strategy in dialogue games. A simple way to explain this connection with game semantics is to interpret the proof $\pi$ as an innocent strategy $\sigma$ in the dialogue category innocent $(\mathscr{X})$ defined in Section II. For notational convenience, we write the associated dialogue games in the same way as the formulas $F$ and $G$. Now, the set of Proponent views of the strategy $\sigma$ may be organized into a tree denoted $\lceil\sigma\rceil$ and called the tree of Proponent views of the strategy $\sigma$. Now, every Proponent view $s$ of the strategy $\sigma$ projects as

- an Opponent view $s \uparrow F$ on the dialogue game $F$,

- a Proponent view $s \uparrow G$ on the dialogue game $G$.

From this follows that the tree $\lceil\sigma\rceil$ induces a pair consisting of a tree of Opponent views $\lceil\sigma\rceil\lceil F$ in the dialogue game $F$ and a tree of Proponent views $\lceil\sigma\rceil \uparrow G$ in the dialogue game $G$. Moreover, every move in the dialogue games $F$ and $G$ appears once (and exactly once) in the tree of views, because the strategy $\sigma$ is total. Hence, the tree $\lceil\sigma\rceil \uparrow F$ contains all the moves of $F$ and the tree $\lceil\sigma\rceil\lceil G$ contains all the moves of $G$. At this point, a good idea is to think of $\lceil\sigma\rceil \uparrow F$ and $\lceil\sigma\rceil \uparrow G$ as dialogue games themselves, rather than trees. The combined fact that $\lceil\sigma\rceil\lceil G$ has the same moves as $G$, and that it is a tree of Proponent views of $G$ leads to the equation:

$$
K_{1}=\lceil\sigma\rceil \uparrow G
$$

and symmetrically for the Opponent views:

$$
J_{1}=\lceil\sigma\rceil \uparrow F .
$$

This justifies to think of the combinators $\kappa_{\otimes}$ and $\kappa_{\otimes}$ as the respective constructors of the Opponent and Proponent views of the strategy $\sigma$. The strategy $\tau$ interpreting the proof between $J_{1}$ and $K_{1}$ is simply the result of the decomposition of the tree of Player views $\lceil\sigma\rceil$ into the two dialogue games $J_{1}$ and $K_{1}$. In particular, every Proponent view of the strategy $\tau$ is a branch in $\lceil\sigma\rceil$, and thus projects as a branch of $J_{1}$ and as a branch of $K_{1}$.

This correspondence between the Opponent-Proponent decomposition of the proof $\pi: F \vdash G$ and the structure of Player views $\lceil\sigma\rceil$ of the associated innocent strategy $\sigma$ is so tight that it is reversible: namely, every innocent strategy $\sigma: F \vdash G$ factors as (9) followed by (10) where $J_{1}$ and $K_{1}$ are defined as the dialogue games:

$$
J_{1}=\lceil\sigma\rceil \uparrow F \quad K_{1}=\lceil\sigma\rceil \uparrow G .
$$

An easy combinatorial argument (by induction) establishes that the two strategies

$$
F \longrightarrow J_{1} \quad K_{1} \longrightarrow G
$$

induced from $\sigma$ are constructed by applying a series of combinators $\kappa_{\otimes}$ and $\kappa_{\otimes}$ respectively, and another easy combinatorial argument (by induction again) that the strategy

$$
J_{1} \longrightarrow K_{1}
$$

decomposes as a sequence of $\boldsymbol{c u t}, \varepsilon$, map, $\eta$ and axiom combinators. This establishes that every functor

$$
\mathcal{F} \quad: \quad \mathscr{X} \longrightarrow \mathscr{D}
$$

to a dialogue category $\mathscr{D}$ may be lifted as a functor

$$
\mathcal{G}: \operatorname{innocent}(\mathscr{X}) \longrightarrow \mathscr{D}
$$

by defining the functor $\mathcal{G}$ on the objects by induction on the dialogue games

- $\mathcal{G}(\neg A)=\neg \mathcal{G}(A)$

- $\mathcal{G}(A \otimes B)=\mathcal{G}(A) \otimes \mathcal{G}(B)$

- $\mathcal{G}(\iota X)=\mathcal{F}(X)$ for every object $X$ of the category $\mathscr{X}$

and on the morphisms by decomposing every innocent strategy $\sigma: A \longrightarrow B$ into a sequence of basic combinators and then 
by transporting each of these combinators to the corresponding combinator in $\mathscr{D}$. This induces a morphism

$$
\mathcal{G}(\sigma): \mathcal{G}(A) \quad \longrightarrow \quad \mathcal{G}(B)
$$

which is uniquely determined because the factorization of an innocent strategy is unique modulo the series of commutative diagrams considered in the proof of Opponent-Proponent factorization. From this follows that the functor $\mathcal{G}$ is properly defined, and is moreover a functor of dialogue categories. From this follows the main result of the paper:

\section{Theorem 5 (Coherence theorem):}

The category innocent $(\mathscr{X})$ of dialogue games and total innocent strategies is equivalent (as a dialogue category) to the free dialogue category free-dialogue $(\mathscr{X})$ generated by the category $\mathscr{X}$.

It should be mentioned at this point that it would be also possible to establish the coherence theorem by more traditional techniques directly adapted from game semantics. The resulting proof would be simpler but much less informative. In particular, the alternative proof would not reveal the relationship between game semantics and string diagrams, nor the algebraic presentation of tensorial proofs by the generators and relations. This combinatorial and algebraic aspect would have remained concealed... Note that, on the other hand, we have recently used this alternative proof technique in order to establish a coherence theorem for a braided notion of dialogue categories, where proofs are manipulated in the same way as topological tangles, see [21], [22] for details.

\section{RElATED WORKS}

The phenomenon of self-adjunction is mentioned by Kock [14] and stressed by Thielecke [30] in his work on continuations. The notion of dialogue category was inspired by the key observation by Hofmann and Streicher [9] and then Selinger [26] that every denotational model of classical logic (that is, of the $\lambda \mu$-calculus) boils down to a continuation model. The formulation of dialogue categories as dialogue chiralities was inspired by Girard's work on polarities in LC [7] and its relationship to dialogue games noticed by Laurent [17], together with the correspondence between games, polarities and continuations originally investigated with Selinger [23]. Note that similar ideas of symmetry appeared independently in the work by Cockett and Seely [4]. The game-theoretic description of the maps $f: X \rightarrow Y$ between atoms in $\mathscr{X}$ as labelled pointers between atomic moves is reminiscent of the game-theoretic account of Girard's geometry of interaction [1] and of proof-nets [2], [25] together with the graphical description of the free ribbon category [13] discussed in the introduction. On the proof-theoretic side, this work should be compared with similar graphical descriptions of the free *-autonomous category [10] and of the free symmetric monoidal closed category [5]. One main difference with the present work is that both constructions [10], [5] require to identify diagrams modulo Trimble's rewiring equalities [3] and thus to depart from the purely topological and diagrammatic notion of proof equality underlying dialogue categories.

\section{REFERENCES}

[1] S. Abramsky, R. Jagadeesan and P. Malacaria. Full Abstraction for PCF In Information and Computation. Vol. 163. 2000.

[2] P. Baillot. Approches dynamiques en sémantique de la logique lineaire: jeux et géometrie de l'interaction. $\mathrm{PhD}$ thesis Universite Aix-Marseille 2, 1999.

[3] R. F. Blute, J. R. B. Cockett, R. A. G. Seely, and T. H. Trimble. Natural deduction and coherence for weakly distributive categories. Journal of Pure and Applied Algebra, 113:229-296, 1996.
[4] R. Cockett, Robert Seely, Polarized category theory, modules, and game semantics, Theory and Applications of Categories 18 (2) (2007) 4-101.

[5] R. Garner, T. Hirschowitz, A. Pardon. Variable binding, symmetric monoidal closed theories, and bigraphs Proceedings of CONCUR 2009, LNCS 5710, Springer Verlag.

[6] J-Y. Girard, Linear logic, Theoretical Computer Science 50 (1987) 1102.

[7] J-Y. Girard, A new constructive logic: Classical logic, Mathematical Structures in Computer Science 1 (3) (1991) 255-296.

[8] M. Hasegawa. A quantum double construction in Rel. To appear in Mathematical Structures in Computer Science.

[9] M. Hofmann, T. Streicher. Completeness of continuation models for $\lambda \mu$ calculus, Information and Computation 179 (2) (2002) 332-355.

[10] D. Hughes. Simple free star-autonomous categories and full coherence. Unpublished manuscript, available on the web page of the author.

[11] M. Hyland and L. Ong, On full abstraction for PCF: I, II and III, Information and Computation 163 (2) (2000) 285-408.

[12] A. Joyal and R. Street. The geometry of tensor calculus, I. Advances in Mathematics 88, 55-112, 1991.

[13] C. Kassel, Quantum Groups, Graduate Texts in Mathematics 155, Springer Verlag, 1995.

[14] A. Kock, On double dualization monads, Mathematica Scandinavica 27 (1970) 151-165.

[15] J. Lambek, From lambda calculus to Cartesian closed categories, in To H. B. Curry: Essays on Combinatory Logic, Lambda Calculus and Formalism, eds. J. P. Seldin and J. Hindley, Academic Press, 1980, pp. 376-402.

[16] J. Lambek, Philip Scott. Introduction to Higher-Order Categorical Logic. Cambridge Studies in Advanced Mathematics. Cambridge University Press 1986.

[17] O. Laurent. Polarized games. Annals of Pure and Applied Logic, number 1-3, volume 130, 79-123, 2004.

[18] G. M. Kelly, S. MacLane. Coherence in closed categories. Journal of Pure and Applied Algebra, 1(1):97-140, 1971.

[19] P-A. Melliès, Asynchronous games 2: the true concurrency of innocence. Theoretical Computer Science, Special Issue "Selected papers of CONCUR 2004", Volume 358, Issues 2-3, pages 200-228, 2006.

[20] P-A. Melliès, Categorical semantics of linear logic, in: Interactive models of computation and program behaviour, Panoramas et Synthèses 27, Société Mathématique de France, 2009.

[21] P-A. Melliès, Braided notions of dialogue categories. Submitted manuscript, available on the web page of the author.

[22] P-A. Melliès, A functorial bridge between proofs and knots. Manuscript, available on the web page of the author.

[23] P-A. Melliès, P. Selinger, Games are continuation models!, talk delivered at Full Completeness and Full Abstraction, Satellite workshop of LICS 2001.

[24] P-A. Melliès, N. Tabareau. Resource modalities in tensor logic. Annals in Pure and Applied Logic, Special Issue edited by Dan Ghica and Russ Harmer, Volume 161, Issue 5, Pages 617-708.

[25] A. Murawski. On Semantic and Type-Theoretic Aspects of PolynomialTime Computations. PhD Thesis, University of Oxford, 2001.

[26] P. Selinger, Control categories and duality: on the categorical semantics of the $\lambda \mu$-calculus, Mathematical Structures in Computer Science 11 (2) (2001) 207-260.

[27] P. Selinger. A survey of graphical languages for monoidal categories. Book chapter. In Bob Coecke, editor, New Structures for Physics, Springer Lecture Notes in Physics 813, pp. 289-355, 2011.

[28] R.H. Street, The formal theory of monads, J. Pure Appl. Alg. 2:149-168, 1972.

[29] R. H. Street. Functorial calculus in monoidal bicategories, Applied Categorical Structures 11 (2003) 219-227

[30] H. Thielecke, Continuation semantics and self-adjointness, Electronic Notes in Theoretical Computer Science 6 (1997) 348-364. 


\section{APPENDIX \\ TENSORIAL LOGIC}

The formulas of tensorial logic are defined by the grammar

$$
A, B \quad:=X|A \otimes B \quad| \quad \mathbf{1}|\neg A| \perp
$$

where $X$ is an atom of the logic. A sequent of tensorial logic is a pair

$$
A_{1}, \ldots, A_{n} \quad \vdash \quad B
$$

consisting of a (possibly empty) sequence of formulas $A_{1}, \ldots, A_{n}$ called the hypothesis of the sequent, and of a (unique) formula $B$ called the conclusion of the sequent. The derivation trees of tensorial logic are constructed by the following rules:

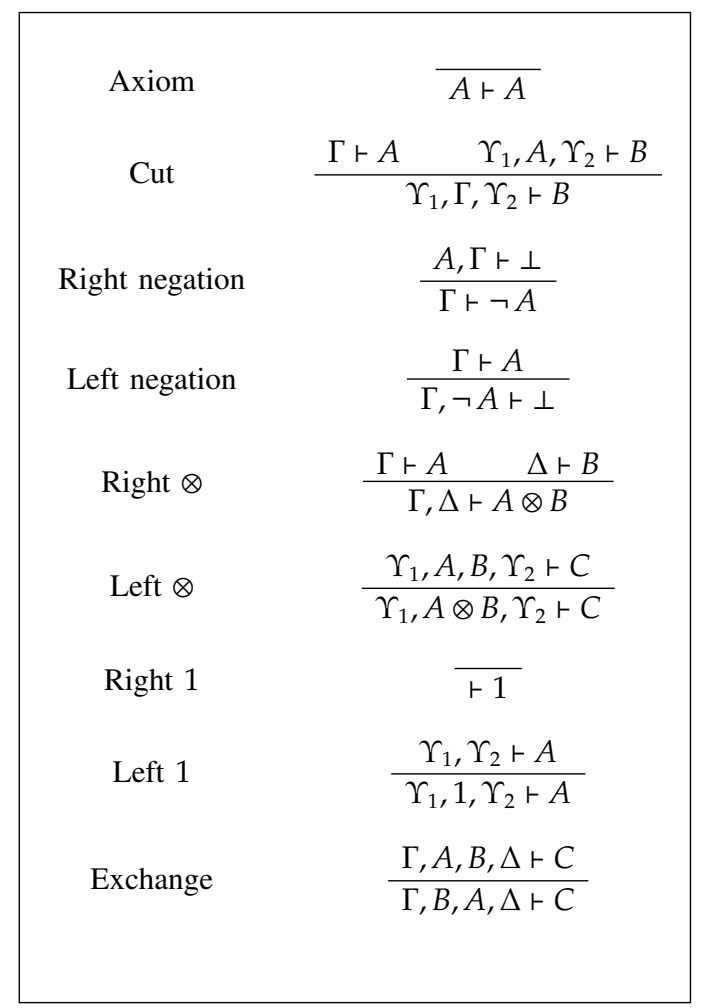

As in the case of minimal logic and of the free cartesian-closed category generated by a given category $\mathscr{X}$, it is natural to extend the logic (in that case, tensorial logic) with a rule:

$$
\operatorname{Map}[f] \quad \bar{X} Y
$$

for each morphism $f: X \rightarrow Y$ of the category $\mathscr{X}$. An equational theory on the derivation trees is then defined in a similar way as in propositional linear logic, see for instance [20]. This equational theory is inherently justified by the fact that it presents the free dialogue category free-dialogue $(\mathscr{X})$ generated by the category $\mathscr{X}$. 